\title{
Protection offered by COVID-19 vaccines in reducing SARS-CoV-2 infection frequency; severity and mortality, among Indian Healthcare Workers: Multicenter, pan-Fortis study
}

\section{Subrat Acharya}

Fortis Escorts Heart Institute

Gaurav Mahindra ( $\sim$ mahindra.gaurav@gmail.com )

Fortis Healthcare

Purushottam Nirala

Fortis Escorts Heart Institute

Sulabh Tripathi

Fortis Healthcare

Bishnu Panigrahi

Fortis Healthcare

Anoop Misra

Fortis C-DOC Hospital

Rahul Pandit

Fortis Hospital Mulund

Manoj Goel

Fortis Memorial Research Institute

S. R. Chowdhury

Fortis Hospital Anandapur

Mrinal Sircar

Fortis Hospital Noida

Vikas Maurya

Fortis Hospital Shalimar Bagh

R. S. Jha

Fortis Escorts Hospital

Avi Kumar

Fortis Escorts Heart Institute

Amit K. Mandal

Fortis Hospital Mohali

Padmakumar Micu

Fortis Hospital Bengaluru 


\section{Ayush Gupta}

Fortis Flt. Lt. Rajan Dhall Hospital

\section{Nagaraj Aithal}

Fortis Hospital Bengaluru

\section{Edel Queen}

Fortis Malar Hospital

\section{Paritosh Baghel}

S.L. Raheja Hospital

\section{Research Article}

Keywords: COVID-19, SARS-CoV-2, Breakthrough infection, Vaccine

Posted Date: November 8th, 2021

DOI: https://doi.org/10.21203/rs.3.rs-1055978/v1

License: (c) (1) This work is licensed under a Creative Commons Attribution 4.0 International License.

Read Full License 


\section{Abstract}

During COVID-19 pandemic, Healthcare Workers (HCWs) were at increased risk for exposure to SARS-CoV2 virus and prioritized for early administration of COVID-19 vaccines in India. Real-life scenario information among vaccinated HCWs acquiring COVID-19 infection, is scarce. We retrospectively analyzed COVID-19 infection frequency, severity, and associated mortality among healthcare workers, immunized with either Covishield or Covaxin vaccines at 27 Fortis Hospitals across 11 Indian states. Positive cases were identified based on RT-PCR or rapid antigen tests for SARS-CoV-2 between 16th January 2021 till 15th May 2021. 20034 HCWs received vaccination. 3971 received 1 dose, 16063 received 2 doses. Post-vaccination, 1139 HCWs acquired COVID-19 infection, 180 (4.53\%) and 959 (5.97\%) among partially and fully vaccinated category, respectively. Breakthrough infection occurred among 913 (5.68\%) HCWs. Concurrently, Case Positivity Rate was 11.9\%, among general population (control). Among 1139 positive cases, mild, moderate, and severe infections were 1059 (93\%), 71 (6.2\%) and $9(0.8 \%)$, respectively with Case Fatality Rate of $0.18 \%$, compared to $0.92 \%$ among general population $(p=0.0043)$. The Case Fatality Rate in vaccinated HCWs was found to be $80 \%$ less than that in general population (control). Hence, COVID-19 vaccines available in India seem to be effective against SARS-CoV-2 virus.

\section{Introduction}

The ongoing COVID-19 pandemic has infected $>232$ million people globally and $>33$ million in India since its origin in December $2019^{1}$. The pandemic is associated with considerable disease mortality, morbidity and economic loss resulting from various control measures needed to limit spread of virus and community transmission. Rapid development of vaccines against the said virus was achieved and immunization programs have been implemented in various countries. In India, two vaccines were licensed for restricted use in emergency situation, based upon phase $2 / 3$ studies. These two vaccines are popularly known as Covishield (AstraZeneca's ChAdOx1 nCoV-19 vaccine (AZD1222) co-invented by the University of Oxford; manufactured by Serum Institute of India) and Covaxin (Bharat Biotech's BBV152 COVID-19 vaccine, developed in collaboration with the Indian Council of Medical Research (ICMR) National Institute of Virology (NIV) ${ }^{2,3}$. The former is a replication-deficient simian adenoviral vector containing full length codon-optimized coding sequence of SARS-CoV-2 spike protein along with a tissue plasminogen activator (tPA) leader sequence. The pooled analysis of 4 randomized trials of the AstraZeneca-Oxford University vaccine reported an overall efficacy of $66.7 \%(95 \% \mathrm{Cl} 57.4 \%-74 \%)$ after 14 days of a two-dose vaccine given at 4-12 weeks interval ${ }^{4}$. Covaxin is an inactivated virus based COVID-19 vaccine, which was developed by treating the virus from Vero cell cultures by beta-propiolactone which deactivate the virus gene while keeping the other viral particle intact. The efficacy of 2 doses of Covaxin at 4-week interval have been claimed to be $78 \%$, based on interim trial result analysis released to press by Bharat Biotech ${ }^{5}$. 
For licensing purposes vaccine efficacy trials need Randomized Control Trial (RCT) data with adequate sample. Such RCTs importantly evaluate three relevant aspects of a vaccine efficacy viz: reduction in infection (sterilizing immunity); reduction in severity of infection (disease modifying immunity); reduction in duration of infection (infection clearing immunity). Such results represent the best-case scenario for assessing vaccine efficacy \& licensing. But vaccine efficacy always does not predict vaccine effectiveness i.e., protection attributed to vaccine administered non-randomly under field conditions. Further, vaccine effectiveness in real life, is not reflected by RCTs restricted to a particular age group, ethnicity, or geographical area. For this reason, studies are needed to assess vaccine effectiveness in reallife scenario after the licensing. Further, the reliability of vaccine effectiveness in real-life scenarios, may be augmented by its evaluation in a cohort of high-risk population who are most likely to contract COVID19 infection, such as active HCWs working during pandemic ${ }^{6,7}$. In such population, the efficacy of a vaccine can be assessed by the frequency of COVID-19 infections detected by RT-PCR and rapid antigen tests on naso-pharyngeal swabs, severity of infection by assessing the number of immunized requiring hospitalization/ oxygen inhalation/ assisted ventilation and resultant mortality rate.

On 16th January 2021, Fortis vaccination program commenced for immunization of HCWs at network hospitals across India. Since February 2021, the 2nd wave of COVID-19 pandemic is active in India, with peak positivity rate (proportion of positive tests to total samples tested), hospitalization rate, ICU admissions and mortalities recorded between 15th April 2021 and 15th June $2021^{8}$. Therefore, a comparison of positivity rate, severity of COVID-19 disease manifestation and mortalities among the vaccinated HCWs and general population (control), may assess the effectiveness of the vaccination strategy adopted by India. Present study describes the COVID-19 infection dynamics among the vaccinated HCWs working at 27 Fortis Hospitals across 11 states in India and the study period included the duration of maximal assault of COVID-19 infection in India.

\section{Methods}

\section{Study Design \& Participants}

It is a retrospective analysis of COVID-19 vaccinated cohort comprising of healthcare workers employed at Fortis Hospitals across 11 states in India. The study period is from $16^{\text {th }}$ January 2021 till $15^{\text {th }}$ May 2021. All healthcare workers who received at least one dose of the vaccine were included in the analysis. The interval between $1^{\text {st }}$ dose and the $2^{\text {nd }}$ dose of COVID-19 vaccine was between 4 to 12 weeks as per prevailing government guidelines. The healthcare workers included in the study comprised of doctors, who were actively treating patients (covid and non-covid), nurses, paramedics, support staff including housekeeping, security, food \& beverage, and administrators regularly coming to hospitals. All participating 27 Fortis Hospitals were providing care services for both Covid as well as non-Covid patients. All healthcare workers included in the study followed covid appropriate behavior, in and outside the hospital. All vaccinated healthcare workers included in the study were neither subjected to RT-PCR/ rapid antigen testing on respiratory specimen nor covid antibody assessment before vaccination. Symptomatic healthcare workers who underwent RT-PCR/ rapid antigen testing post-vaccination and 
found to be positive, were diagnosed to have COVID-19 infection. For the vaccinated healthcare workers acquiring COVID-19 infection, demographic data and details related to their hospital admission, oxygen requirement, assisted ventilation and morality were retrieved from the Fortis Hospital data base, maintained for vaccination purpose. Details on COVID-19 disease manifestation, and mortalities among the general population (control) were collected for comparison. In relation to the study period, the vaccine frequency of two doses of the licensed vaccine amongst Indian population did not exceed $3 \%{ }^{8}$. (Total share $=10.13 \%$; share of people fully vaccinated $=2.9 \%$; share of people partially vaccinated $=7.2 \%$ ). Thus, nearly $97 \%$ of the general population in India were nonvaccinated during study period and served as the control population in the present study.

For each hospital, approvals were obtained from their respective Ethics Committee and the study was registered with Clinical Trials Registry-India (CTRI). (CTRI registration number CTRI/2021/10/037376; "Fortis Registry Study for Vaccinated Healthcare Workers infected with SARS-CoV-2/ COVID-19").

\section{Data Source and Management}

Each hospital made use of a standardized questionnaire and clinical record format to maintain employee demographic data and vaccine related information. While corresponding information for control population was sought from reliable online sources ${ }^{9,10}$. Information on severity of symptoms among personnel and mortality after one and both doses of vaccine were obtained separately from hospital records. The event day or date of COVID-19 positive test report (RT-PCR/ rapid antigen testing) was retrieved to assess the total covid positivity frequencies as well as breakthrough infection among the vaccinee cohorts.

\section{Study Variables}

Covid-19 positive cases were identified as those detected with SARS-CoV-2 RNA or antigen in a respiratory specimen on RT-PCR or rapid antigen testing. Breakthrough infections were cases with detection of SARS-CoV-2 RNA or antigen in a respiratory specimen collected from a person $\geq 14$ days after they have completed all recommended doses of COVID-19 vaccine ${ }^{11}$. Classification of mild, moderate, and severe covid infection was defined by following criteria ${ }^{12}$ :

\section{Mild infection}

Upper respiratory tract symptoms like cough, sore throat \&/or fever without shortness of breath or hypoxia $\left(\mathrm{SpO}_{2}>94 \%\right.$ after 6 -minute walk).

Moderate infection as, any one of:

1. Respiratory rate $\geq 24$ / minute, breathlessness

2. $\mathrm{SpO}_{2}$ : $90 \%$ to $\leq 93 \%$ on room air (or after 6 -minute walk is below $94 \%$ requiring oxygen support through nasal prong/ masks to maintain $\mathrm{SpO}_{2}$ above $94 \%$ ). 
Severe Infection as, any one of:

1. Respiratory rate $>30 /$ minute, breathlessness

2. $\mathrm{SpO}_{2}<90 \%$ on room air (needing ICU care, assisted ventilation).

Any oxygen inhalation to maintain $\mathrm{SpO}_{2}$ above $94 \%$ was considered as oxygen requirement in hospitalized healthcare workers.

Assisted ventilation refers to an individual needing CPAP, BiPAP or intubation using a mechanical respirator.

Symptoms like sore throat, headache, cough, breathlessness, myalgia, anosmia, ageusia, diarrhea, nausea, vomiting, abdominal pain, itchy patch on body part were recorded for Covid positive individuals among the vaccinated cohort.

Follow-up: The Covid-19 vaccination program was instituted from $16^{\text {th }}$ January 2021 , at all 27 Fortis hospitals included in this study. The cutoff date (for censuring data) was $15^{\text {th }}$ May 2021. All healthcare workers, who received the Covid vaccine and whose data was retrievable were included in the study.

\section{OUTCOME MEASURES}

Primary outcome measures: To assess COVID-19 mortality rate among vaccinated HCWs in comparison to the general population (control) during the COVID-19 pandemic.

Secondary outcome measures: To assess COVID-19 infection frequency among vaccinated HCWs and evaluate the severity of disease in the said group.

Statistics analysis: Categorical variable are expressed as number (percentage) and comparison between groups was performed with Chi-square test (Fisher's exact test), as applicable. Continuous variables are expressed as Mean \pm Standard Deviation. A p-value $<0.05$ was considered statistically significant. All statistical analyses were performed using the SPSS software (SPSS Version 25).

\section{Results}

The 27 sites included in the study (Table 1) had 26040 personnel of whom 20034 were administered vaccines, either the scheduled two doses of vaccine $(n=16063: 80.2 \%)$ or one dose $(n=3971: 19.8 \%)$. The remaining 6006 employees were migratory/ contract personnel whose vaccination data was not available and were therefore excluded from the study. Table 1. enlists the hospitals, distribution of healthcare workers, and the frequency of vaccine doses administered to them. Doctors, Nurses, Paramedics (including support staff) and Administrators constituted 18\% ( $n=4696), 26 \%(n=6769), 47 \%(n=12239)$ and $9 \%(n=2336)$ of HCWs, respectively. Ninety percent of the HCWs included in the study received Covishield vaccine (AstraZeneca's ChAdOx1 nCoV-19 vaccine (AZD1222) and remaining 10\% of the vaccinated HCW cohort received Covaxin (Bharat Biotech's BBV152 COVID-19 vaccine 
During the study period, a total of 1139 (5.68\%) of the 20034 vaccinated HCWs were reported to be positive for SARS-CoV-2 (RT-PCR or rapid antigen testing on nasopharyngeal swab). After one dose of vaccination ( $\mathrm{n=3971)} 180$ (4.53\%) (Table 2) had COVID-19 infection before they received the second dose and after both the doses ( $\mathrm{n}=16063), 959$ (5.97\%) (Table 2) had infection. Breakthrough infection ( $\geq 14$ days after both doses of vaccination) was documented in 913 (5.68\%) (Table 2) of the vaccinated cohort, indicating that 46 of the fully vaccinated group had COVID-19 infection after the second dose but within 14 days of the last dose of vaccination.

As explained in Table 3, the mean age for Doctors/ Nurses/ Paramedics (technicians/ housekeeping/ security)/ Administrators was found to be 43/ 31/ 36/ 37 years respectively.

During the same study period the COVID-19 case positivity rate among the general population in 11 states $(n=79,13,53,000)$ where the 27 Fortis hospitals exist was found to be around $11.92 \%$ (Table 4). Apparently, the infection rate amongst the vaccinated HCWs seemed markedly lower. We did not statistically compare the above two groups, because the population infection data indicated case positivity rate (number of positive tests/total number of tests done), whereas being a retrospective analysis the present study did not calculate case positivity rate among the vaccinated HCWs but, included those who were symptomatic and got tested. The total vaccinated cohort were not tested prospectively for COVID -19, thereby the actual case positivity rate was not available for them. Despite this lacuna, it seems that vaccinated HCWs had lower rate of infection. Among the infected HCWs $(\mathrm{N}=1139)$, the proportion of Doctors, Nurses, Paramedics and Administrators were 23\% $(n=262), 43.4 \%$ $(n=495), 15.7 \%(n=179)$ and $17.8 \%(n=203)$ respectively. When the proportion of infected HCWs were assessed among the total staff under each category, it was seen that the frequencies of infection among doctors, nurses and paramedics were 5.5\% (262/4696), 7.3\% (495/6769), and 1.5\% (179/12239). This would indicate that post vaccination, COVID-19 infection may be higher among the nurses as compared to doctors and other paramedics.

Among the vaccinated COVID-19 infected HCWs ( $N=1139), 93 \%(n=1059), 6.2 \%(n=71)$, and $0.8 \%(n=9)$ had mild, moderate (only oxygen required through nasal prong/ mask) and severe (ICU admission, assisted ventilation) COVID-19 infection respectively (Table 8$)$. Two patients died (0.18\%). One patient died after one dose of the vaccination and the other died after 14 days of receiving both doses of vaccination. Because more than $90 \%$ were mild infection the distribution of the degree of severity was not markedly different across the age/gender or categories of the staffs. During the same study period the case fatality rate among all the COVID-19 positive cases $(n=94,31,527)$ in the control population (infected population in the 11 states where Fortis hospitals exist), was $0.92 \%$ ( $n=86,627)$ (Table 9). The mortality rate among the vaccinated and infected HCWs was found to be $80 \%$ lower than the control population $(0.18 \%$ vs $0.92 \% ; p=0.0043$; fisher's exact test) which is a significant finding.

The mean time interval, between last vaccine dose and positive test report was 47 days, 50 days, and 52 days for partially vaccinated, fully vaccinated and breakthrough cases, respectively (Table 10). 


\section{Discussion}

The present study provides the dynamics of COVID-19 infection among vaccinated HCWs in tertiary care settings across 27 Fortis hospitals in India. Despite being a retrospective, descriptive analysis, the study reveals three important aspects of COVID-19 vaccine in a group of immunized, high-risk individuals (HCWs), namely:

1. It seems, COVID-19 infection rate amongst vaccinated HCWs was markedly lower as compared to control population.

2. Disease manifestation amongst the vaccinated HCWs was found to be largely mild in $93 \%$ cases; with moderate symptoms in $6 \%$ cases while severe symptoms were manifested in just $0.8 \%$ cases, needing ICU care/ assisted ventilation.

3. The case fatality rate amongst the high-risk group of HCWs was found to be $80 \%$ lower as compared to control population. Nearly $90 \%$ of the HCWs included in the study received Covishield vaccine [(AstraZeneca's ChAdOX1 nCoV-19 vaccine (AZD1222)] while remaining 10\% of the vaccinated HCW cohort received Covaxin (Bharat Biotech's BBV152 COVID-19 vaccine,) developed in collaboration with the Indian Council of Medical Research (ICMR) - National Institute of Virology (NIV).

The present study did not analyze or compare the two vaccine groups with each other since proportion of Covishield administered doses was $\sim 90 \%$ and heavily skewed in favor of one group. Therefore, any comparison seemed redundant.

The above-mentioned vaccines are reported to have a protective efficacy of 67 to $78 \%$. The present study documented a significantly lower rate of infection amongst the vaccinated HCWs who were actively working during the pandemic, at a time when the infection frequencies were reported to be amongst the highest. As mentioned in result section, an infection rate varying between 4.5-5.97\% among the vaccinated $\mathrm{HCW}$ s was markedly lower than the case positivity rate of $\sim 12 \%$ during the same period, observed in the control population of 11 Indian states where Fortis hospitals exist (Table 2, 4). However, as described in result section, the COVID-19 infection rate amongst HCWs reported in the present study were not case positivity rate because the number of total vaccinated HCWs were not tested for presence of COVID-19 infection prospectively and the reported frequencies among this group was derived from overtly symptomatic HCWs who were tested. Despite this short coming the markedly lower rate of COVID infection among the vaccinated cohort of HCWs may not be ignored and provides an important observation.

Amongst the infected cohort of vaccinated HCWs, the clinical manifestation was mild in $>90 \%$ cases. Severe clinical manifestation indicated by ICU care/ assisted ventilation was less than $1 \%$ and only $0.18 \%$ among the infected cohort of HCWs died due to the disease. During the study period the infected control population (population in the 11 states where Fortis hospitals exist) had a mortality rate of $0.92 \%$ which was significantly higher than the mortality rate of $0.18 \%$ amongst HCWs $(p=0.0043)$ (Table 9$)$, thereby indicating a reduction of mortality rate by nearly $80 \%$ after vaccination. Indeed, ICMR (Indian Council of 
Medical Research) reported that 2 doses of COVID-19 vaccine prevented death by nearly $98 \%{ }^{13,14}$. However, reduction in mortality rates among high-risk groups post-vaccination, assessed during peak of pandemic, using locally available vaccines has not been reported earlier. Therefore, the present results would assure the medical fraternity all over the country about the benefits of COVID-19 vaccines that are locally available in India. The above-mentioned benefit was observed across all categories of Fortis employees.

A recent publication evaluated effectiveness of COVID-19 m-RNA Vaccine BNT162b2 vaccine (PfizerBioNTech) and mRNA-1273 vaccine (Moderna) ${ }^{15}$. The study was conducted across 25 U.S. states, representing more than 500,000 health care personnel. Vaccine effectiveness was evaluated by subjecting immunized healthcare personnel to RT-PCR or rapid antigen testing for SARS-CoV-2. Participants who tested positive for SARS-CoV-2 were identified as cases $(n=1482)$ while those who tested negative were identified as controls. After complete vaccination, vaccine effectiveness was $88.8 \%$ $(95 \% \mathrm{Cl}, 84.6$ to 91.8$)$ and $96.3 \%(95 \% \mathrm{Cl}, 91.3$ to 98.4$)$, respectively. Vaccine protection was documented across all age groups and even those with comorbidities. However, the study did not evaluate any possible reduction in mortality rate.

The present study has several lacunae. It, being a retrospective study, could not assess true case positivity rate among the vaccinated HCW cohort, because the vaccinated HCWs were neither tested for COVID-19 infection before vaccination nor were they tested after vaccination. Those who had symptoms and tested positive for COVID-19 were the ones available for further analysis. The study could not compare the severity of clinical manifestation among the HCWs and control population due to nonavailability of severity data among the infected control population. The information on comorbidity among the vaccinated cohort was not available and therefore influence of comorbidities on COVID-19 infection after vaccination could not be ascertained. Since $>90 \%$ vaccinated cohort had mild infection, the influence of advanced age and other known factors associated with severity of illness could not be studied. Importantly, the adoption of COVID-19 precautionary measures by HCWs, such as, use of Personal Protective Equipment (PPE) and access to repeated sanitization/ disinfection safety measures, could have been instrumental in increasing overall vaccine effectiveness and safety.

However, the strength of the study was evaluation of vaccine effectiveness in a real-life scenario at 27 sites across 11 Indian states, amongst high-risk group of individuals being continuously exposed to COVID-19 infection.

In conclusion, the study demonstrates that the COVID-19 vaccine, locally available in India, could offer substantial benefits to high-risk individuals such as Healthcare Workers by reducing frequency of infections, lowering severity of illness, and significantly reducing mortality rate once infected.

\section{Declarations}

Acknowledgments: Source of support is Fortis Healthcare Limited 


\section{Conflict of Interest: None}

Disclaimer: The findings and conclusions in this article are those of the authors and do not necessarily represent the official position of Fortis Healthcare Limited. This study is for academic purposes only. It does not make any commercial claim nor does the study endorse any vaccine.

\section{References}

1. The Johns Hopkins University \& Medicine. Coronavirus Resource Center (CRC) COVID-19 dashboard. https://coronavirus.jhu.edu/map.html (Accessed September 28, 2021).

2. AstraZeneca Media Centre. Press release, Serum Institute of India obtains emergency use authorization in India for AstraZeneca's COVID-19 vaccine. https://www.astrazeneca.com/mediacentre/press-releases/2021/serum-institute-of-india-obtains-emergency-use-authorisation-in-indiafor-astrazenecas-covid-19-vaccine.html (Accessed April 1, 2021).

3. Bharat Biotech products. Vaccines. https://www.bharatbiotech.com/covaxin.html (Accessed September 28, 2021).

4. Voysey M, Costa Clemens SA, Madhi SA, et al. Single-dose administration and the influence of the timing of the booster dose on immunogenicity and efficacy of ChAdOx1 nCoV-19 (AZD1222) vaccine: a pooled analysis of four randomized trials [published correction appears in Lancet. 2021 Mar 6;397(10277):880]. Lancet. 2021;397(10277):881-891. doi:10.1016/S0140-6736(21)00432-3.

5. Raches Ella, Siddarth Reddy, William Blackwelder, Varsha Potdar, Pragya Yadav, Vamshi Sarangi, et al. Efficacy, safety, and lot to lot immunogenicity of an inactivated SARS-CoV-2 vaccine (BBV152): a, double-blind, randomized, controlled phase 3 trial. The Covaxin study group. medRxiv 2021.06.30.21259439. https://doi.org/10.1101/2021.06.30.21259439.

6. Nguyen LH, Drew DA, Joshi AD, et al. Risk of COVID-19 among frontline healthcare workers and the general community: a prospective cohort study. Preprint. medRxiv. 2020;2020.04.29.20084111. Published 2020 May 25. doi:10.1101/2020.04.29.20084111.

7. Gómez-Ochoa SA, Franco OH, Rojas LZ, et al. COVID-19 in Health-Care Workers: A Living Systematic Review and Meta-Analysis of Prevalence, Risk Factors, Clinical Characteristics, and Outcomes [published correction appears in Am J Epidemiol. 2021 Jan 4;190(1):187]. Am J Epidemiol. 2021;190(1):161-175. doi:10.1093/aje/kwaa191.

8. Hannah Ritchie, Edouard Mathieu, Lucas Rodés-Guirao, Cameron Appel, Charlie Giattino, Esteban Ortiz-Ospina, et al. (2020) - "Coronavirus Pandemic (COVID-19)". Published online at OurWorldInData.org. Retrieved from: 'https://ourworldindata.org/coronavirus' [Online Resource].

9. Association for India's Development. Indian states: Weekly positive/Weekly tested. https://aidindia.org/daily-covid-positive-rates/ (Accessed September 1, 2021).

10. NDTV Coronavirus Outbreak Live Statistics. https://www.ndtv.com/coronavirus (Accessed September 1, 2021). 
11. Centers for Disease Control and Prevention. COVID-19 Vaccine Breakthrough Case Investigation and Reporting. https://www.cdc.gov/vaccines/covid-19/health-departments/breakthrough-cases.html (Accessed September 1, 2021).

12. AlIMS/ICMR-COVID-19 National Task Force/ Joint Monitoring Group (Dte.GHS), Ministry of Health \& Family Welfare, Government of India. Clinical guidance for management of Adult Covid-19 patients. https://covid.aiims.edu/clinical-guidance-for-management-of-adult-covid-19-patients/ (Accessed May 20, 2021). https://www.icmr.gov.in/pdf/covid/techdoc/COVID_Management_Algorithm_17052021.pdf (Accessed May 20, 2021).

13. Press Information Bureau; Ministry of Information \& Broadcasting; Government of India. ICMR Delhi; Bhargava B; Protective Effect of Vaccines Against Death. https://twitter.com/COVIDNewsByMIB/status/1435923051011473408?s=20 (Accessed September 9, 2021).

14. The Free Press Journal. COVID-19: First shot $96.6 \%$ effective in preventing death, $97.5 \%$ for 2 doses. https:// www.freepressjournal.in/india/covid-19-first-shot-966-effective-in-preventing-death-975-for-2doses-says-center (Accessed September 10, 2021).

15. Pilishvili T, Gierke R, Fleming-Dutra KE, et al. Effectiveness of mRNA Covid-19 Vaccine among U.S. Health Care Personnel [published online ahead of print, 2021 Sep 22]. N Engl J Med. 2021;10.1056/NEJMoa2106599. doi:10.1056/NEJMoa2106599.

\section{Tables}




\begin{tabular}{|c|c|c|c|c|c|c|c|}
\hline $\begin{array}{l}\text { Fortis Hospital } \\
\text { (State) }\end{array}$ & Doctors & Nurses & Paramedics* & Admin & $\begin{array}{l}\text { Total } \\
\text { Staff }\end{array}$ & $\begin{array}{c}\text { Staff received } \\
\text { Dose } 1 \\
\end{array}$ & $\begin{array}{c}\text { Staff received Dose } \\
1 \& 2\end{array}$ \\
\hline Kangra (HP) & 35 & 93 & 148 & 62 & 338 & 266 & 209 \\
\hline Amritsar (Punjab) & 87 & 247 & 503 & 125 & 962 & 892 & 836 \\
\hline Ludhiana (Punjab) & 87 & 254 & 356 & 134 & 831 & 541 & 435 \\
\hline Mohali (Punjab) & 352 & 698 & 993 & 344 & 2377 & 1557 & 1312 \\
\hline Anandapur (W. Bengal) & 298 & 494 & 480 & 199 & 1471 & 1411 & 1002 \\
\hline FHKI \& FMC (W. Bengal) & 78 & 74 & 137 & 78 & 367 & 300 & 157 \\
\hline CDOC (Delhi) & 68 & 24 & 46 & 32 & 170 & 120 & 98 \\
\hline FEHI (Delhi) & 288 & 590 & 901 & 211 & 1990 & 1717 & 1230 \\
\hline Vasant Kunj (Delhi) & 178 & 238 & 302 & 169 & 887 & 799 & 644 \\
\hline Shalimar Bagh (Delhi) & 404 & 567 & 786 & 203 & 1960 & 1452 & 1076 \\
\hline La Femme (Delhi) & 79 & 81 & 111 & 72 & 343 & 280 & 250 \\
\hline FMRI (Haryana) & 393 & 476 & 1001 & 555 & 2425 & 1604 & 1580 \\
\hline Faridabad (Haryana) & 107 & 283 & 651 & 36 & 1077 & 1064 & 898 \\
\hline Noida (Uttar Pradesh) & 316 & 358 & 459 & 336 & 1469 & 1153 & 662 \\
\hline Raigarh (Chhattisgarh) & 26 & 71 & 122 & 53 & 272 & 261 & 253 \\
\hline Jaipur (Rajasthan) & 257 & 304 & 442 & 178 & 1181 & 784 & 288 \\
\hline Kalyan (Maharashtra) & 30 & 77 & 117 & 63 & 287 & 280 & 255 \\
\hline Mulund (Maharashtra) & 373 & 463 & 622 & 262 & 1720 & 1281 & 1247 \\
\hline SL Raheja (Maharashtra) & 203 & 232 & 451 & 95 & 981 & 838 & 730 \\
\hline Vashi (Maharashtra) & 239 & 134 & 373 & 103 & 849 & 731 & 647 \\
\hline $\begin{array}{l}\text { Bannerghatta Road } \\
\text { (Karnataka) }\end{array}$ & 396 & 442 & 741 & 275 & 1854 & 1266 & 1143 \\
\hline $\begin{array}{l}\text { Cunningham Road } \\
\text { (Karnataka) }\end{array}$ & 90 & 177 & 367 & 25 & 659 & 315 & 220 \\
\hline Nagarbhavi (Karnataka) & 60 & 69 & 116 & 18 & 263 & 189 & 111 \\
\hline Rajajinagar (Karnataka) & 39 & 56 & 104 & 11 & 210 & 180 & 170 \\
\hline La Femme (Karnataka) & 42 & 41 & 40 & 46 & 169 & 134 & 86 \\
\hline Malar (Tamil Nadu) & 143 & 170 & 181 & 159 & 653 & 500 & 440 \\
\hline Vadapalani (Tamil Nadu) & 38 & 56 & 128 & 53 & 275 & 119 & 84 \\
\hline Total & $\begin{array}{l}4696 \\
(18 \%)\end{array}$ & $\begin{array}{l}6769 \\
(26 \%)\end{array}$ & $\begin{array}{l}12239 \\
(47 \%)\end{array}$ & $\begin{array}{l}2336 \\
(9 \%)\end{array}$ & 26040 & $\begin{array}{l}20034 \\
(77 \%)\end{array}$ & $\begin{array}{l}16063 \\
(62 \%)\end{array}$ \\
\hline
\end{tabular}

*Includes Technicians; Housekeeping; Security etc.

Table 2. COVID-19 infection frequencies amongst Vaccinate Healthcare Workers (HCWs)

\begin{tabular}{lccc} 
Criteria & Vaccinated HCWs & COVID-19 positive HCWs & COVID-19 positive HCWs (\%) \\
\hline HCWs who have received Dose 1 only & 3971 & 180 & $4.53 \%$ \\
HCWs who have received both Dose 1 and 2 & 16063 & 959 & $5.97 \%$ \\
Breakthrough cases* $(\geq 14$ days after Dose 2) & 16063 & 913 & $5.68 \%$ \\
\hline
\end{tabular}

*Breakthrough cases are a subset of Fully Vaccinated cohort 


\begin{tabular}{|c|c|c|c|c|c|c|}
\hline Group & Age & Doctors & Nurses & Paramedics & Administrators & Total \\
\hline $\mathrm{PV}^{*}$ & Mean \pm SD (Range) & $39 \pm 11(26-69)$ & $29 \pm 8(22-60)$ & $35 \pm 8(22-56)$ & $34 \pm 7(24-51)$ & $33 \pm 9(22-69)$ \\
\hline $\mathrm{FV}^{\dagger}$ & Mean \pm SD (Range) & $44 \pm 11(23-79)$ & $31 \pm 8(21-63)$ & $36 \pm 9(20-62)$ & $37 \pm 8(23-59)$ & $36 \pm 10(20-79)$ \\
\hline $\mathrm{BT}^{+}$ & Mean \pm SD (Range) & $44 \pm 11(23-79)$ & $31 \pm 8(21-63)$ & $37 \pm 9(20-62)$ & $37 \pm 8(23-59)$ & $36 \pm 10(20-79)$ \\
\hline Total & Mean \pm SD (Range) & $43 \pm 11(23-79)$ & $31 \pm 8(21-63)$ & $36 \pm 9(20-62)$ & $37 \pm 8(23-59)$ & $36 \pm 10(20-79)$ \\
\hline Group & Gender & Doctors & Nurses & Paramedics & Administrators & Total \\
\hline PV & Male/ Female & 22-Dec & $25 / 74$ & 17-Mar & $14 / 13$ & $78 / 102$ \\
\hline FV & Male / Female & $145 / 83$ & $79 / 317$ & $116 / 43$ & $102 / 74$ & $442 / 517$ \\
\hline BT & Male/ Female & $139 / 75$ & $77 / 308$ & $108 / 40$ & $99 / 67$ & $423 / 490$ \\
\hline Total & Male/ Female & $167 / 95$ & $104 / 391$ & $133 / 46$ & $116 / 87$ & $520 / 619$ \\
\hline \multicolumn{7}{|c|}{$\begin{array}{l}\text { *Partially vaccinated: Beneficiaries who have received Dose } 1 \text { only } \\
\text { †Fully vaccinated: Beneficiaries who have received both Doses } 1 \text { and } 2 \\
\text { ¥ Breakthrough cases: COVID-19 positive } \geq 14 \text { days after completing both Doses } 1 \text { and } 2\end{array}$} \\
\hline
\end{tabular}

\begin{tabular}{|c|c|c|c|c|c|c|}
\hline Period & $\begin{array}{l}\text { Population in Indian States with Fortis } \\
\text { presence (11 states) }\end{array}$ & $\begin{array}{l}\text { New } \\
\text { cases }\end{array}$ & $\begin{array}{l}\text { Tests } \\
\text { conducted }\end{array}$ & $\begin{array}{l}\text { Case Positivity } \\
\text { Rate (CPR) }\end{array}$ & Mortalities & $\begin{array}{c}\text { Case } \\
\text { Fatality } \\
\text { Rate (CFR) }\end{array}$ \\
\hline $\begin{array}{l}16 \text { Jan } 21 \text { to } 16 \\
\text { Mar } 21\end{array}$ & $79,13,53,000$ & $5,18,551$ & $2,52,13,487$ & $2.06 \%$ & 4,941 & $0.95 \%$ \\
\hline $\begin{array}{l}17 \text { Mar } 21 \text { to } \\
15 \text { May } 21\end{array}$ & $79,13,53,000$ & $89,12,976$ & $5,38,99,155$ & $16.54 \%$ & 81,686 & $0.92 \%$ \\
\hline $\begin{array}{l}\text { 16 Jan } 21 \text { to } 15 \\
\text { May } 21\end{array}$ & $79,13,53,000$ & $94,31,527$ & $7,91,12,642$ & $11.92 \%$ & 86,627 & $0.92 \%$ \\
\hline
\end{tabular}

\begin{tabular}{lccccc}
\hline Table 5. Manifestation of COVID-19 symptoms among Partially Vaccinated HCWs (Dose 1 only) & Severe symptoms \\
\hline $\begin{array}{l}\text { Partially } \\
\text { Vaccinated }\end{array}$ & $\begin{array}{c}\text { COVID-19 } \\
\text { cases }\end{array}$ & $\begin{array}{c}\text { Mild } \\
\text { symptoms }\end{array}$ & $\begin{array}{c}\text { Moderate } \\
\text { symptoms }\end{array}$ & $\begin{array}{c}\text { Mssisted } \\
\text { ventilation }\end{array}$ \\
\hline Doctors & & & 0 & 0 & 0 \\
Nurse & 34 & 30 & 4 & 1 & 0 \\
Paramedics & 99 & 94 & 1 & 0 & 0 \\
\hline Administrators & 20 & 19 & 2 & 0 & 0 \\
\hline Total & 27 & 25 & $11(6.11 \%)$ & $1(0.55 \%)$ & 0 \\
\hline$*$ Brought Dead & 180 & $168(93.33 \%)$ & & 0 \\
\hline
\end{tabular}




\begin{tabular}{|c|c|c|c|c|c|c|}
\hline \multirow{2}{*}{$\begin{array}{l}\text { Fully } \\
\text { Vaccinated }\end{array}$} & \multirow[t]{2}{*}{ COVID-19 cases } & \multirow[t]{2}{*}{ Mild symptoms } & \multirow{2}{*}{$\begin{array}{c}\text { Moderate symptoms } \\
\text { Oxygen required }\end{array}$} & \multicolumn{2}{|c|}{ Severe symptoms } & \multirow[t]{2}{*}{ Mortality } \\
\hline & & & & ICU admission & Assisted ventilation & \\
\hline Doctors & 228 & 200 & 24 & 3 & 1 & 0 \\
\hline Nurse & 396 & 370 & 25 & 1 & 0 & 0 \\
\hline Paramedics & 159 & 153 & 4 & 1 & 1 & $1 *$ \\
\hline Administrators & 176 & 168 & 7 & 1 & 0 & 0 \\
\hline Total & 959 & $891(92.91 \%)$ & $60(6.25 \%)$ & $6(0.62 \%)$ & $2(0.21 \%)$ & 1 \\
\hline
\end{tabular}

\begin{tabular}{|c|c|c|c|c|c|c|}
\hline \multirow{2}{*}{$\begin{array}{l}\text { Breakthrough } \\
\text { cases }\end{array}$} & \multirow{2}{*}{$\begin{array}{l}\text { COVID-19 } \\
\text { cases }\end{array}$} & \multirow{2}{*}{$\begin{array}{c}\text { Mild } \\
\text { symptoms }\end{array}$} & \multirow{2}{*}{$\begin{array}{c}\begin{array}{c}\text { Moderate } \\
\text { symptoms }\end{array} \\
\text { Oxygen required }\end{array}$} & \multicolumn{2}{|c|}{ Severe symptoms } & \multirow[t]{2}{*}{ Mortality } \\
\hline & & & & $\begin{array}{c}\text { ICU } \\
\text { admission } \\
\end{array}$ & $\begin{array}{c}\text { Assisted } \\
\text { ventilation }\end{array}$ & \\
\hline Doctors & 214 & 187 & 23 & 3 & 1 & 0 \\
\hline Nurse & 385 & 361 & 23 & 1 & 0 & 0 \\
\hline Paramedics & 148 & 143 & 3 & 1 & 1 & $1 *$ \\
\hline Administrators & 166 & 158 & 7 & 1 & 0 & 0 \\
\hline Total & 913 & 849 (92.99\%) & $56(6.13 \%)$ & $6(0.66 \%)$ & $2(0.22 \%)$ & 1 \\
\hline
\end{tabular}

Table 8. Manifestation of COVID-19 symptoms among all vaccinated HCWs

\begin{tabular}{|c|c|c|c|c|c|c|}
\hline \multirow{2}{*}{$\begin{array}{l}\text { Vaccinated } \\
\text { cohort } \\
\text { (Partially + } \\
\text { Fully) }\end{array}$} & \multirow[t]{2}{*}{ COVID-19 cases } & \multirow[t]{2}{*}{ Mild symptoms } & \multirow{2}{*}{$\begin{array}{c}\text { Moderate symptoms } \\
\text { Oxygen required }\end{array}$} & \multicolumn{2}{|c|}{ Severe symptoms } & \multirow[t]{2}{*}{ Mortality } \\
\hline & & & & ICU admission & Assisted ventilation & \\
\hline Doctors & 262 & 230 & 28 & 3 & 1 & 0 \\
\hline Nurse & 495 & 464 & 29 & 2 & 0 & 1 \\
\hline Paramedics & 179 & 172 & 5 & 1 & 1 & 1 \\
\hline Administrators & 203 & 193 & 9 & 1 & 0 & 0 \\
\hline Total & 1139 & 1059 (93\%) & $71(6.2 \%)$ & $7(0.6 \%)$ & $2(0.2 \%)$ & 2 \\
\hline
\end{tabular}




\begin{tabular}{|c|c|c|c|c|}
\hline $\begin{array}{l}\text { Study period } \\
16^{\text {th }} \text { Jan } 21 \text { to } 15^{\text {th }} \text { May } 21\end{array}$ & Population & COVID-19 cases & Mortalities & Case Fatality Rate \\
\hline Partially vaccinated Fortis HCWs & 3,971 & 180 & 1 & $0.55 \%$ \\
\hline Fully vaccinated Fortis HCWs & 16,063 & 959 & 1 & $0.10 \%$ \\
\hline Total vaccinated Fortis HCW cohort & 20,034 & 1,139 & 2 & $0.18 \% *$ \\
\hline $\begin{array}{l}\text { Control population } \dagger \\
\text { (11 states which have Fortis presence) }\end{array}$ & $79,13,53,000$ & $94,31,527$ & 86,627 & $0.92 \% *$ \\
\hline India† & $1,39,00,06,000$ & $1,41,20,000$ & $1,18,010$ & $0.84 \%$ \\
\hline
\end{tabular}

\begin{tabular}{lccc}
\hline \multicolumn{3}{l}{ Table 10. Time interval between last vaccine dose and positive test report (days) } \\
\hline Group & Mean & Standard Deviation & Range \\
\hline Partially vaccinated (180 cases) & 47 & 32 & 0 to 116 \\
Fully vaccinated (959 cases) & 50 & 18 & 1 to 88 \\
\hline Breakthrough cases (913 cases) & 52 & 15 & 14 to 74 \\
\hline Total (1139 cases) & 49 & 21 & 0 to 116 \\
\hline
\end{tabular}

